\title{
Parameter Free Hierarchical Graph-Based Clustering for Analyzing Continuous Word Embeddings
}

\author{
Thomas A. Trost Dietrich Klakow \\ Saarland University \\ Saarbrücken, Germany \\ \{thomas.trost, dietrich.klakow\}@lsv.uni-saarland.de
}

\begin{abstract}
Word embeddings are high-dimensional vector representations of words and are thus difficult to interpret. In order to deal with this, we introduce an unsupervised parameter free method for creating a hierarchical graphical clustering of the full ensemble of word vectors and show that this structure is a geometrically meaningful representation of the original relations between the words. This newly obtained representation can be used for better understanding and thus improving the embedding algorithm and exhibits semantic meaning, so it can also be utilized in a variety of language processing tasks like categorization or measuring similarity.
\end{abstract}

\section{Introduction}

There are different ways to assess word embeddings (Yaghoobzadeh and Schütze, 2016). While some authors focus on general properties, as for example Levy et al. (2015) or Hashimoto et al. (2016), most evaluations are with respect to specific tasks. Examples of the latter include the works by Baroni et al. (2014), Schnabel et al. (2015), or Rothe and Schütze (2016), to name but a few. The objective of this paper is to introduce a method for getting a grasp of the global structure of embeddings, which is different from general schemes for dimensionality reduction like t-SNE (Maaten and Hinton, 2008), the methods summarized by Van Der Maaten et al. (2009), or visualization interfaces such as Roleo (Sayeed et al., 2016) and GoWvis (Tixier et al., 2016). The method presented here is a specific way of clustering (a field nicely reviewed by Jain et al. (1999)) that works particularly well for the current objective.
We present a global analysis of the statistical properties of the embedding space. This is based on the output of the well-known word2 vec program (Mikolov et al., 2013), using the example of the dataset published alongside the source code on the web ${ }^{1}$, which was generated with the skipgram model with negative sampling. This dataset was trained on parts of the English Google news corpus and consists of 3,000,000 words with 300dimensional embedding vectors. First, densities in the embedding space will be explored. Based on that a parameter free hierarchical graph-based clustering approach is developed that is the basis of a tool that allows to explore the neighborhood of a term of interest.

The paper is structured as follows: After a quick discussion of statistical properties of the dataset, the concept of the graphical neighborhood hierarchy is explained. Specific properties of the resulting graphs are brought into the context of peculiarities of the dataset for showing that this representation is particularly well-suited. Finally, the semantic properties of the graphs are briefly evaluated.

\section{Properties of Embedding Spaces}

First, a look at global statistics of the dataset lays a basis for justifying later choices and interpreting the hierarchy. Herein, special care must be taken with respect to effects of the high dimensionality.

The distribution of the values of single vector components all look very similar and peak clearly at the origin, but they exhibit relatively heavy tails. The distribution of the $L_{2}$-norm ${ }^{2}$ of the embedding vectors can be seen in fig. 1, both for all and rare words, where the latter are those that are not found

\footnotetext{
${ }^{1}$ https://code.google.com/archive/p/ word2vec/

${ }^{2}$ For a discussion of the choice of distance function, see section 3.2 below.
} 
in the 1 billion word corpus (Chelba et al., 2013). Even though the curves show a drop at the origin

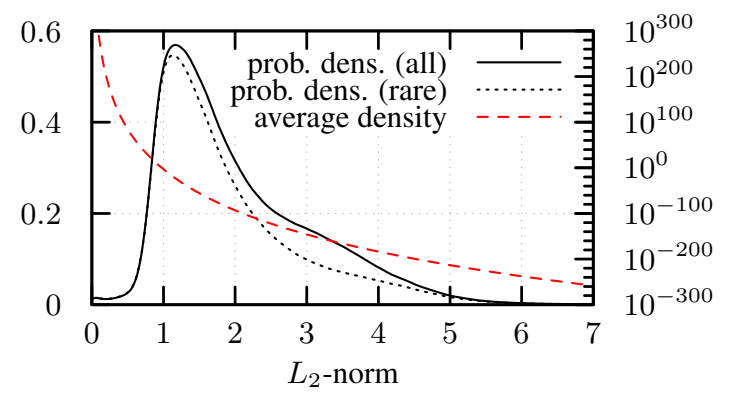

Figure 1: Probability densities for finding a word vector with the given norm, for all and rare words (left axis, density for rare words rescaled for reflecting proportion) and plot of the resulting average density at the respective distance to the origin (right axis).

and a clear peak at slightly above one, they are mostly a consequence of the high dimensionality of the embedding space. This becomes apparent in the plot of the actual average density (words per volume) at a given distance from the origin (also fig. 1), which decreases very rapidly and monotonically. It can be concluded that embedding vectors are highly concentrated around the origin, but that common words tend to lie at an intermediate distance to the origin and do not fully follow the general distribution.

Next, a principle component analysis can be done in order to evaluate how isotropic the dataset actually is. It reveals that the largest and smallest eigenvalues are only about an order of magnitude apart and that the top 20 percent of eigenvalues account for roughly 50 percent of the total variance in the dataset. While this is clearly not fully isotropic, there appear to be no directions that are completely superfluous. For the global picture, approximate isotropy is thus a fairly reasonable assumption.

To complete the general statistical exploration of the embedding space we want to look at specific word classes (common nouns, verbs and adjectives) versus other words that belong to none of these classes. We also want to explore the impact of the word frequency on the position in the embedding space. Figure 2 gives the results. The first - however non-surprising - observation is that the center of the embedding space is made up of low frequency words that are not nouns, verbs or adjectives. These three POS classes densely popu- late the surface of a 300 dimensional sphere in a distance of three to four from the center of the embedding space. Exploring this rim in more detail is most interesting for applications. For this we will develop a parameter free method to study the vicinity of a word of interest to the user of the tool.

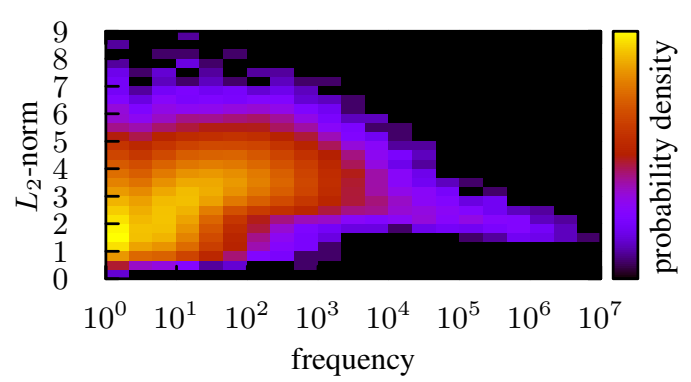

(a) all words

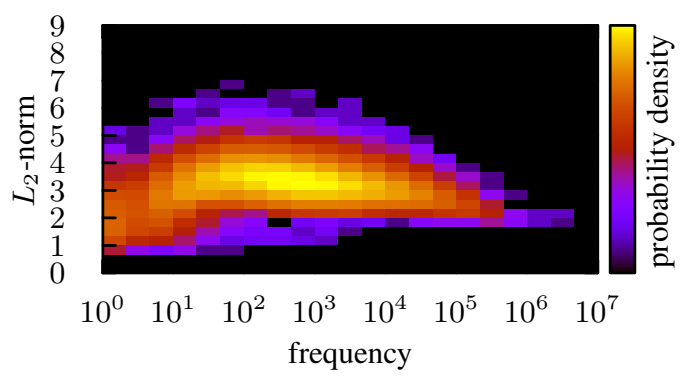

(b) common nouns, verbs, and adjectives

Figure 2: Probability density for finding an embedding vector of a word of given frequency and a given $L_{2}$-norm and thus distance from the origin. Note that the density is given in log-scale.

\section{Nearest Neighbor Graph}

Consider a set of embedding vectors $\mathcal{W}$ that is equipped with a distance function $d: \mathcal{W} \times \mathcal{W} \rightarrow$ $\mathbb{R}_{0}^{+}$. The nearest neighbor graph (NNG) on $\mathcal{W}$ is a directed weighted graph where each vertex $v$ has outdegree one and is connected to its nearest neighbor $w=\arg \min _{w^{\prime}} d\left(v, w^{\prime}\right)$, with the weight corresponding to the distance. In case of ambiguity, the nearest neighbor has to be selected via additional criteria or randomly. Note that the nearest neighbor relation need not be reciprocal. The $k$-NNG which incorporates the notion of $k$ nearest neighbors can be defined in a similar way, but it lacks most of the nice properties of the simple NNG, some of which will be discussed next.

Naive implementations for nearest neighbor search scale quadratically with the number $n=$ $|\mathcal{W}|$ of nodes, however, $\mathcal{O}(n \log n)$-solutions are available (Sankaranarayanan et al., 2007), whose 
efficiency depends on the dimensionality of $\mathcal{W}$. Thus, in particular for high-dimensional spaces, approximate nearest neighbor search may be much more efficient (Muja and Lowe, 2009).

\subsection{Clusters}

Here, the weakly connected components of an NNG are denoted as clusters. That is to say, there is a path between every two vertices within a cluster, if the direction of the edges is ignored. It can readily be seen that each cluster must have exactly one cluster root, which is a pair of vertices that see each other as their nearest neighbor. Apart from that, there cannot be any cycles in a cluster, so it can be considered as two trees each of which is rooted in one vertex of the cluster root. This treelike and very clear structure of the clusters makes them interesting for our purposes. Example clusters extracted from the NNG of the word2vec dataset are depicted in fig. 5, which will be discussed below.

\subsection{Choice of Distance Function}

The particular choice of a distance function $d$ may drastically affect the form of an NNG. In general, it is advantageous if $d$ has the properties of an actual metric, because then it corresponds closely to the human notion of a distance which makes it easier to interpret the results.

For a variety of additional reasons, here, the classical Euclidean distance

$$
d_{\mathrm{E}}(v, w):=\sqrt{\sum_{i}\left(v_{i}-w_{i}\right)^{2}}
$$

is chosen. Most importantly, $d_{\mathrm{E}}$ is invariant under orthogonal transformations (rotating and flipping), which goes well with the apparent isotropy of the embedding space. With this distance function, no particular component or direction is given more attention than another. Besides that, the Euclidean distance is relatively cheap and easy to compute and there is a lot of literature on specialized methods for finding NNGs with this metric. Furthermore, $d_{\mathrm{E}}$ is loosely related to the cosine similarity that is used as the main ingredient during the training of the embedding mapping.

\section{Neighborhood Hierarchy}

By means of an NNG, the local structure between the words within each of its clusters can be understood fairly well, but any information about the relationship between different clusters is completely lost. In order to deal with that, the simple NNG can be extended via a neighborhood hierarchy $(\mathrm{NH})$, which adds information about the neighborhood relation between clusters, clusters of cluster and so on. A sketch of the first two levels of such a hierarchy is given in fig. 3. Each cluster is equipped with what could be called a macro vertex, which might for example be the mean of the vertices in the cluster, the center of the cluster root, or the most frequent (and thus hopefully most important) word in the cluster. Then the NNG of the macro vertices can be determined. This leads to new clusters, new macro vertices, another NNG and so forth, till the top level is reached, which contains only one cluster of macro vertices. In order to make the whole hierarchy browsable, the macro vertices can be given a clearer meaning by assigning one representative word to each of them. This word might for example be the nearest one to the macro vertex or the most frequent word in the cluster.

While the nearest neighbor relationship alone is somewhat problematic, as small changes in the dataset may result in huge differences in the cluster layout (in particular in high-dimensional spaces), the hierarchy smooths this effect away to some degree, as lower-level flipping between clusters will probably not affect higher level clusters.

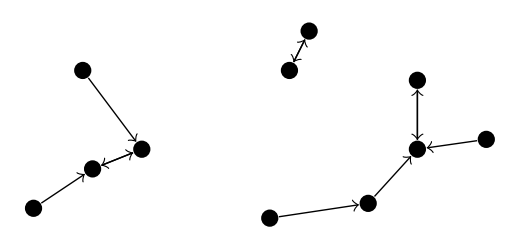

(a) 1st level (words)

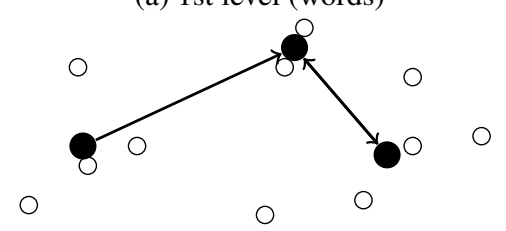

(b) 2nd level (macro vertices)

Figure 3: Sketch of a cluster hierarchy. The massive dots are the centers of mass of the clusters of small dots and form a cluster themselves.

\section{Hierarchy of Vector Embeddings}

The method from the previous section can now be applied to the set of embedding vectors. The word vectors form clusters and the macro vertices introduced above can be seen as generic or in some 
way paraphrasing terms for the words in their cluster (see section 6 for semantic evaluation), under the given premise that similar words are mapped to nearby vectors. The $\mathrm{NH}$ produces a partitioning of the vector space in the spirit of a Voronoi diagram at various levels of coarseness and can thus be used to navigate through the otherwise hard to grasp high-dimensional space.

\subsection{General Properties of the Hierarchy}

The NH of the word2vec dataset has a total of six levels. The first level contains the words themselves, higher levels comprise macro vertices as described above. General properties of the graphs on the different levels are given in table 1 . In accordance with the hierarchical structure, the number of words and thus the number of clusters decrease exponentially.

Typical characteristics of the graphs are strongly influenced by the fact that the graphs are NNGs. As each cluster has one root and each of the $n$ vertices has out-degree one, the reciprocity

$$
r:=\frac{\text { \#reciprocal edges }}{n}
$$

is proportional to the inverse of the average number of words per cluster. The more elaborate measure of reciprocity $\rho$ introduced by Garlaschelli and Loffredo (2004) reduces to

$$
\rho=\frac{r(n-1)-1}{n-2} \underset{n \gg 1}{\approx} r
$$

and is thus almost the same as $r$ for the larger graphs. Note that the expression (3) is not defined for the sixth level. $\rho$ is rather low compared to other natural networks, but interestingly it lies just in the range of other word networks (Garlaschelli and Loffredo, 2004).

Here, the depth $d$ of the graphs for a specific leaf is the number of edges between the leaf and the respective cluster root. The average of $d$ over all leafs and the maximum value of $d$ are presented in table 1 . While $\max (d)$ decreases exponentially, possibly in accordance with the shrinking of the cluster size, particularly the constancy of the midlevel $\varnothing d$ is striking and a sign of two contrary processes. The longer connections on the lower levels are compensated for by more small connections, or, in other words, the smaller high-level clusters are more regular in terms of their depths.

On all levels, the NNGs appear to be scale free (Barabási and Bonabeau, 2003), with the con-

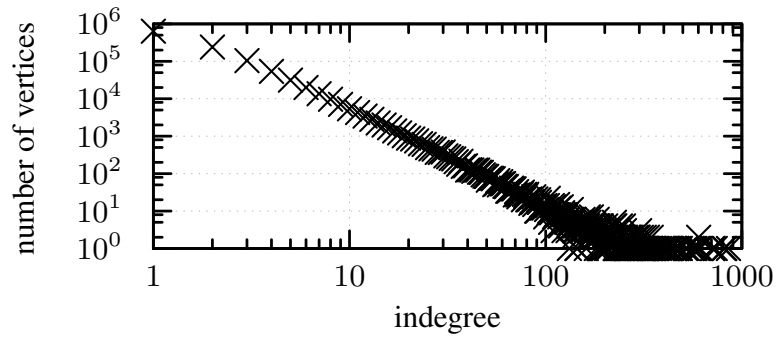

Figure 4: Log-log scatter plot of the number of times a first-level vertex has a particular indegree. While this point cannot be represented in the chart, there are about $1.8 \times 10^{6}$ vertices with an indegree of 0 in the NNG.

\begin{tabular}{lcccccc}
\hline level & 1 & 2 & 3 & 4 & 5 & 6 \\
\hline \# words & $3 \cdot 10^{6}$ & 99,884 & 6750 & 540 & 55 & 2 \\
\# clusters & 99,884 & 6750 & 540 & 55 & 2 & 1 \\
$\varnothing$ w./cl. & 30.0 & 14.8 & 12.5 & 9.8 & 27.5 & 2.0 \\
$r$ & 0.067 & 0.14 & 0.16 & 0.20 & 0.073 & 1.0 \\
$\rho$ & 0.067 & 0.14 & 0.16 & 0.20 & 0.055 & - \\
$\varnothing d$ & 6.6 & 2.5 & 2.5 & 2.5 & 2.4 & - \\
$\max (d)$ & 25 & 16 & 10 & 6 & 4 & - \\
\hline
\end{tabular}

Table 1: General properties of the $\mathrm{NH}$ of the word2vec dataset. In the third row, the average number of words per cluster is given. See section 5.1 for definitions of the other quantities.

straint that the higher-level graphs contain too little vertices for making a definite statement about that. Exemplarily, this feature can be seen for the first-level graph in fig. 4. Scale freeness is primarily associated to processes in which new vertices are attached preferably to those existing vertices that already have a large indegree. In the current context this sheds a light on the behavior of the learning algorithm, specifically because scale freeness is encountered on all levels. A possible interpretation is that the algorithm leads to a multi-level attaching of words and groups of words while trying to put similar words as close to each other as possible. Interestingly, different semantic networks exhibit the scale-free property, too (Steyvers and Tenenbaum, 2005).

\subsection{Examples of Clusters}

Examples of first-level clusters extracted from the word2 vec dataset can be found in fig. 5. At this point, only the surface can be scratched, because there are thousands of such clusters and many of them are interesting in some way.

The dataset contains a pretty raw set of words; 


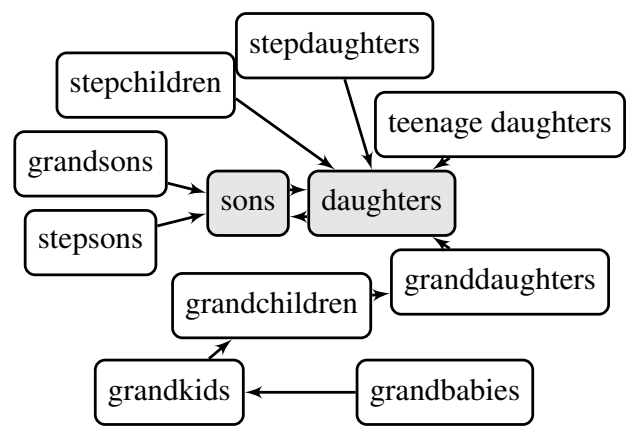

(a) Cluster below "sons"

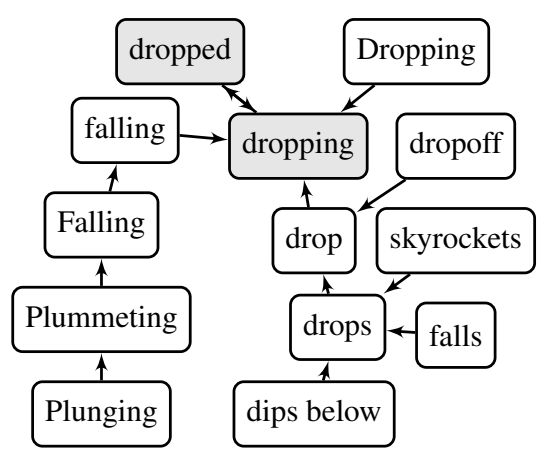

(b) Cluster below "dropped"

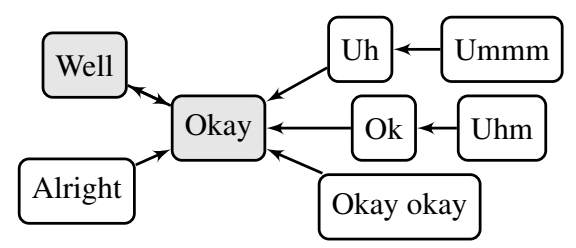

(c) Cluster below "Well"

Figure 5: Example clusters from actual dataset, with cluster roots marked gray. The most frequent word of the cluster is chosen as the macro vertex and given in the description.

proper names, capitalized or inflected words, misspellings, or fillers have not been stripped from the data. From the context-based training method (Mikolov et al., 2013) it can be expected that syntactically similar words end up close to each other, which is indeed seen in the $\mathrm{NH}$, as in fig. 5c, where fillers and certain discourse items, all of them capitalized, form a cluster. This might also explain that only plural forms have gathered in fig. 5a. While this often means that connected items are also semantically similar, antonyms like "drops" and "skyrockets" in fig. 5b are frequently close to each other due to their similar syntactic roles. Despite such problems, it must be stressed that fig. 5 is not the result of extensive cherry picking, but that semantically meaningful clusters are the rule, even if the large number of proper names and more or less meaningless padding words sometimes shadow the more interesting clusters.

After this glance at some first-level clusters, an example of the actual hierarchy is shown in fig. 6 . On the lowest levels, the words are closely related to their neighbors and the words in their parent clusters, just as it has been the case in fig. 5 . This is still the case on the next levels, but, in general, the higher one gets in the hierarchy, the looser the connection to the words on the lower levels, because a lot of words are collected beneath a specific high-level word and not all of them can be equally suitable. In the specific situation in fig. 6 , the words on the third level are mostly related to finance and economy and the same accounts for the fourth level, with more and more rather unspecific words in between. Revealing this is just what the hierarchy is good for: The fact that "index"-related words are collected in the "financial region" of the embedding space is not self-evident. If the embeddings would not have been trained on a news corpus but on scientific resources, the position of the word "index" would very likely be a different one.

Here, the primary purpose of the $\mathrm{NH}$ is getting a better understanding of embeddings and the meaning of the relations in the $\mathrm{NH}$ must therefore not be over-interpreted, because they explicitly have to be left as unaltered as possible for making them good representatives of the raw dataset. Specific relations can often (see below) but not necessarily be transferred into a semantic order, as can exemplarily be seen in fig. 5a, where kinship relations are not organized as one would probably put them. However, this is what the dataset looks like in terms of geometrical neighborhood. If certain words are positioned in a different way from what could be expected, this does not mean that the clustering went wrong, but rather that something interesting happened in the embedding space.

\subsection{Geometry of Clusters}

The neighborhood relation gives a good view of the relative positioning of the words, but the geometry of the clusters and their orientation in the vector space is mostly veiled. Luckily, certain statistics reveal that there is much regularity in the shape of the clusters, so that the cluster alone contains enough information for telling where a specific word is likely to be found.

For each pair of embedding vector $v$ and the 


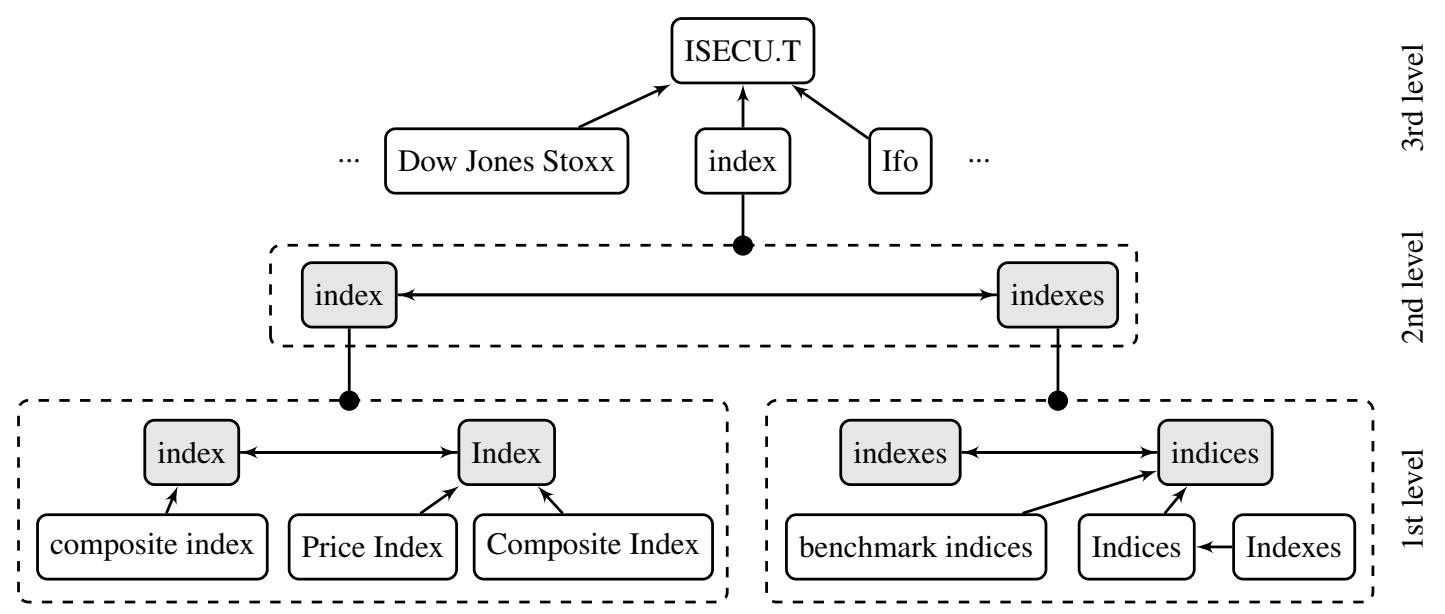

Figure 6: Example of relations between clusters on the three lowest levels of the hierarchy. The dashed boxes frame clusters. Note that only an excerpt of the (much larger) cluster on the third level is shown. Lines ending in a circle indicate the connection between macro vertices and their clusters.

respective nearest neighbor $w$, the radiality $R \in$ $[-1,1]$ of this nearest neighbor relation can be defined as the normalized scalar product between $v$ and the difference vector between $w$ and $v$ via

$$
R:=\frac{v \cdot(w-v)}{|v||w-v|} .
$$

Positive values of $R$ mean, that $w$ lies farther away from the origin than $v$, while negative values imply the opposite. In fig. 7, the probability density for finding a certain value for $R$ is shown. It can be

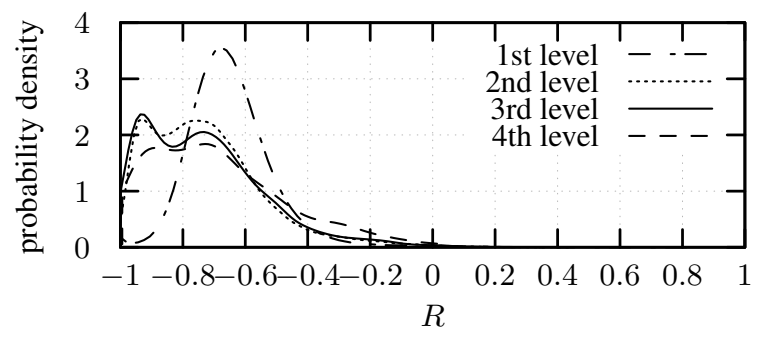

Figure 7: Radiality $R$, as defined in (4).

concluded that for the data at hand, the neighborhood relation on all levels strongly tends to point "inward", i.e. towards the origin of the embedding space. In other words, it is almost certain, that the nearest neighbor of a word vector lies closer to the origin of the coordinate system than the word vector itself. On this basis and as the clusters are basically trees that grow away from the cluster root, it can be expected that the cluster roots typically lie near to the origin, compared to the other vertices in the respective cluster. This can be checked by plotting the probability density for finding a cluster with a given percentage of vertices that are farther away from the origin than the cluster root (Figure 8). As expected, in most clusters the ma-

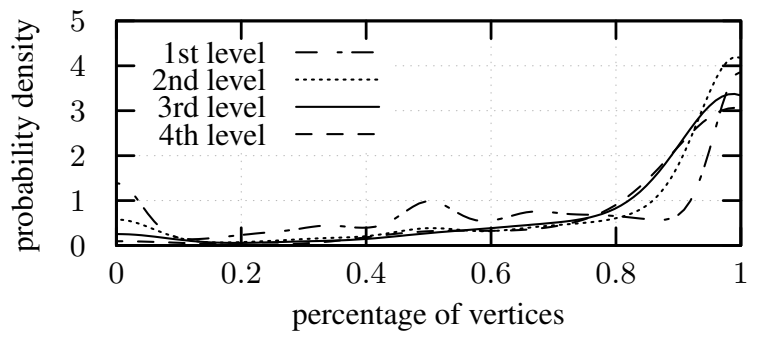

Figure 8: Probability density for finding clusters where the given percentage of vertices lie farther away from the origin than the cluster root.

jority of vertices tends to lie farther outside than the cluster root. Nevertheless, the probability density shows little bumps around fractions of small integers like $\frac{1}{3}, \frac{1}{2}$, or $\frac{2}{3}$. These are mostly due to small clusters, for which the position of the cluster root within the cluster seems to be less predictable. However, these clusters contain only a small fraction of all words and their structure is easy to understand anyway. If only relatively large clusters are taken into account, the probability density peaks much more strongly around the value 1 .

Taking all this into account, and even though there is no notion of geometry in the NNG, the meaning of clusters like those in fig. 5 becomes much more transparent: The root is very likely the closest vertex to the origin and the other vertices are successively farther outside. For example, the representation vectors of the words "falling", 
"Falling", "Plummeting", and "Plunging" have an increasing $L_{2}$-norm or distance from the origin and they form a chain in the graph in fig. 5b. Only a bit additional information about the position of the root is thus sufficient for getting an idea of the position and orientation of the whole cluster.

\section{Evaluation}

The focus of this paper is on the analysis of embeddings. Nevertheless, as already mentioned above, the findings presented in the previous sections indicate that the NH might be used for NLP tasks beyond visualization of word embeddings or other large high-dimensional datasets, because the neighborhood and macro vertex relations appear to be connected to semantical relations between the words, particularly on the lower levels. Possible tasks that directly come to mind are measuring relatedness or similarity, various kinds of tagging, and classification. In contrast to typical semantical frameworks like WordNet (Miller, 1995) or FrameNet (Baker et al., 1998) whose creation requires extensive human resources, the $\mathrm{NH}$ can be created without expert knowledge in a very short time and has the capability of including much more words.

Zesch and Gurevych (2007) analyze graphs extracted from Wikipedia ${ }^{3}$ and summarize a variety of methods for evaluating semantical relations. In this spirit and for a first and quick quantitative view at the $\mathrm{NH}$, similarity between neighbors in the graph and between words and their macro vertex are tested by calculating the respective $\mathrm{Wu}$ Palmer similarity scores (Wu and Palmer, 1994) on WordNet (Miller, 1995). Other scores basically lead to similar results and are thus not discussed in more detail. Because the number of words in WordNet is much smaller than that in the dataset under consideration, the analysis is limited to those words that can be found in both datasets, which amounts to 54,586 words. For that to be possible, a NH of these words alone is used, which is distinct from the full hierarchy discussed above. The usefulness of these results for a much smaller dataset can be justified by envisioning that the sparser NNG must roughly be a skeleton of the full graph for geometrical reasons and must thus be related to the latter. Besides that, quantifying similarity on the smaller graph is interesting in its own right.

\footnotetext{
${ }^{3}$ http://www.wikipedia.org
}

The results for the first four levels of the $\mathrm{NH}$ are shown in fig. 9. Intuitively, the semantic relations between neighbors or words and macro vertices are expected to be stronger, if more "probability mass" can be found on the right side of the plot, because then more relations correspond to a higher similarity. In order to clarify the meaning of the curves, a baseline curve is added that corresponds to an equivalent evaluation of random word pairs.

Both the neighborhood relation and the macro vertex assignment yield noticeably better results than the baseline. In accordance with earlier remarks, the curves confirm that the semantical significance of the hierarchy is much higher on the lower levels. While the first and the second level appear to exhibit a large amount of meaningful relations, the higher levels are not much better than the baseline.

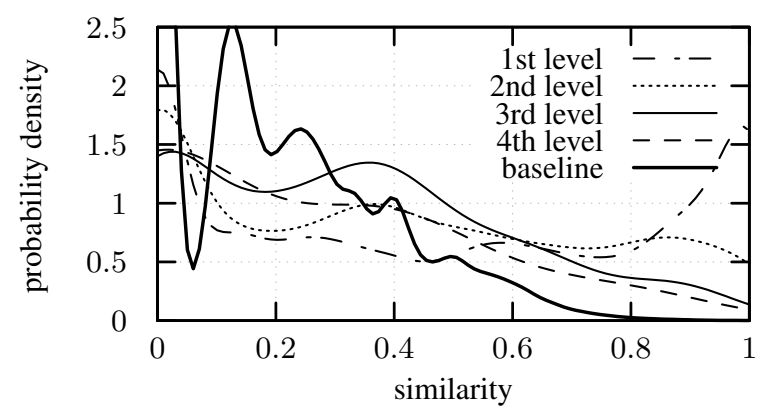

(a) Similarity between words and their neighbors

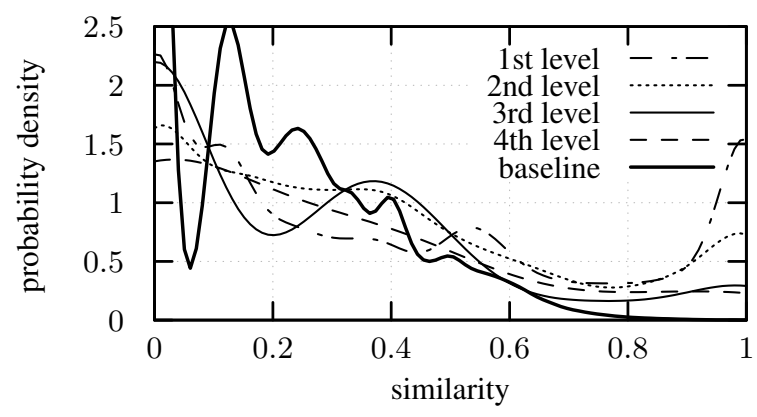

(b) Similarity between words and their macro vertices

Figure 9: Evaluation of similarity. The curves represent the probability density of finding a certain Wu-Palmer similarity between the respective words. The baseline peaks at $(0,6.8)$ but is cut off for clarity of the other curves.

\section{Conclusion and Outlook}

In this paper we have presented a general graphbased method for the analysis of embedding spaces. At the heart lies a neighborhood hier- 
archy $(\mathrm{NH})$, a parameter free, flexible and general concept for clustering data in arbitrary spaces, which eliminates the problem of interpreting highdimensional vectors while preserving the most important geometric information. In order to get a better understanding of the data, a variety of statistical properties of word embeddings has been evaluated. First evidence of the semantic significance of the $\mathrm{NH}$ has been established by relating it to WordNet data.

This method of analysis will allow researchers to interactively explore the neighborhood relations in an embedding space. This will enable them not only to get a better intuition of the structure of embedding spaces but will also give them new ideas on how to incorporate embeddings in natural language processing tasks like information extraction or other tasks that require semantic knowledge.

\section{Acknowledgments}

This work was funded by the Deutsche Forschungsgemeinschaft (DFG) under grant SFB 1102.

\section{References}

Collin F. Baker, Charles J. Fillmore, and John B. Lowe. 1998. The Berkeley FrameNet Project. In Proceedings of the 36th Annual Meeting of the Association for Computational Linguistics and 17th International Conference on Computational Linguistics, Volume 1. Association for Computational Linguistics, Montreal, Quebec, Canada, pages 86-90. https://doi.org/10.3115/980845.980860.

Albert-László Barabási and Eric Bonabeau. 2003. Scale-free networks. Scientific American 288(5):60-69. https://doi.org/10.1038/scientificamerican0503-60.

Marco Baroni, Georgiana Dinu, and Germán Kruszewski. 2014. Don't count, predict! A systematic comparison of context-counting vs. context-predicting semantic vectors. In Proceedings of the 52nd Annual Meeting of the Association for Computational Linguistics (Volume 1: Long Papers). Association for Computational Linguistics, Baltimore, Maryland, pages 238-247. http://www.aclweb.org/anthology/P14-1023.

Ciprian Chelba, Tomas Mikolov, Mike Schuster, Qi Ge, Thorsten Brants, and Phillipp Koehn. 2013. One Billion Word Benchmark for Measuring Progress in Statistical Language Modeling. CoRR abs/1312.3005. http://arxiv.org/abs/1312.3005.

Diego Garlaschelli and Maria I. Loffredo. 2004. Patterns of Link Reciprocity in Directed
Networks. Physical Review Letters 93(26). https://doi.org/10.1103/physrevlett.93.268701.

Tatsunori Hashimoto, David Alvarez-Melis, and Tommi Jaakkola. 2016. Word Embeddings as Metric Recovery in Semantic Spaces. Transactions of the Association for Computational Linguistics 4:273-286. https://transacl.org/ojs/index.php/tacl/article/view/809.

Anil K. Jain, M. Narasimha Murty, and Patrick J. Flynn. 1999. Data Clustering: A Review. ACM computing surveys (CSUR) 31(3):264-323. https://doi.org/10.1145/331499.331504.

Omer Levy, Yoav Goldberg, and Ido Dagan. 2015. Improving distributional similarity with lessons learned from word embeddings. Transactions of the Association for Computational Linguistics 3:211-225. https://transacl.org/ojs/index.php/tacl/article/view/570.

Laurens van der Maaten and Geoffrey Hinton. 2008. Visualizing data using t-SNE. Journal of Machine Learning Research 9(Nov):2579-2605.

Tomas Mikolov, Ilya Sutskever, Kai Chen, Greg S Corrado, and Jeff Dean. 2013. Distributed representations of words and phrases and their compositionality. In Advances in Neural Information Processing Systems. pages 3111-3119.

George A. Miller. $1995 . \quad$ WordNet: A Lexical Database for English. Communications of the ACM 38(11):39-41. https://doi.org/10.1145/219717.219748.

Marius Muja and David G. Lowe. 2009. Fast approximate nearest neighbors with automatic algorithm configuration. Proceedings of the Conference on Computer Vision Theory and Applications (VISAPP) (1) 2(331-340):2. https://doi.org/10.1.1.160.1721.

Sascha Rothe and Hinrich Schütze. 2016. Word Embedding Calculus in Meaningful Ultradense Subspaces. In Proceedings of the 54th Annual Meeting of the Association for Computational Linguistics (Volume 2: Short Papers). Association for Computational Linguistics, Berlin, Germany, pages 512-517. http://anthology.aclweb.org/P16-2083.

Jagan Sankaranarayanan, Hanan Samet, and Amitabh Varshney. 2007. A fast all nearest neighbor algorithm for applications involving large pointclouds. Computers \& Graphics 31(2):157-174. https://doi.org/10.1016/j.cag.2006.11.011.

Asad Sayeed, Xudong Hong, and Vera Demberg. 2016. Roleo: Visualising thematic fit spaces on the web. In Proceedings of ACL-2016 System Demonstrations. Association for Computational Linguistics, Berlin, Germany, pages 139-144. http://anthology.aclweb.org/P16-4024. 
Tobias Schnabel, Igor Labutov, David Mimno, and Thorsten Joachims. 2015. Evaluation methods for unsupervised word embeddings. In Proceedings of the 2015 Conference on Empirical Methods in Natural Language Processing. Association for Computational Linguistics, Lisbon, Portugal, pages 298-307. http://aclweb.org/anthology/D15-1036.

Mark Steyvers and Joshua B. Tenenbaum. 2005. The Large-scale structure of semantic networks: Statistical analyses and a model of semantic growth. $\mathrm{Cog}$ nitive Science 29(1):41-78.

Antoine Tixier, Konstantinos Skianis, and Michalis Vazirgiannis. 2016. GoWvis: A Web Application for Graph-of-Words-based Text Visualization and Summarization. In Proceedings of ACL-2016 System Demonstrations. Association for Computational Linguistics, Berlin, Germany, pages 151-156. http://anthology.aclweb.org/P16-4026.

Laurens Van Der Maaten, Eric Postma, and Jaap Van den Herik. 2009. Dimensionality Reduction: A Comparative Review. Journal of Machine Learning Research 10:66-71.

Zhibiao Wu and Martha Palmer. 1994. Verb Semantics and Lexical Selection. In Proceedings of the 32nd Annual Meeting of the Association for Computational Linguistics. Association for Computational Linguistics, Las Cruces, New Mexico, USA, pages 133-138. https://doi.org/10.3115/981732.981751.

Yadollah Yaghoobzadeh and Hinrich Schütze. 2016. Intrinsic Subspace Evaluation of Word Embedding Representations. In Proceedings of the 54th Annual Meeting of the Association for Computational Linguistics (Volume 1: Long Papers). Association for Computational Linguistics, Berlin, Germany, pages 236-246. http://www.aclweb.org/anthology/P161023.

Torsten Zesch and Iryna Gurevych. 2007. Analysis of the Wikipedia category graph for NLP applications. In Proceedings of the TextGraphs-2 Workshop (NAACL-HLT 2007). pages 1-8. 